\title{
REIMAGINING AND DEMOCRATISING THE NEW PRODUCT DEVELOPMENT PROCESS THROUGH A BOARD GAME ANALOGY
}

\author{
Michael O'SULLIVAN and Con SHEAHAN \\ University of Limerick, Ireland
}

\begin{abstract}
Researchers have spent decades developing tools and techniques to aid teams in the new product development (NPD) process. Despite this, studies have shown that there is a huge gap between their academic prevalence and their industry adoption. For the fuzzy front-end in particular, there is a wide range of tools to choose from, including the Kano Model, the House of Quality, and many others. In fact, there are so many tools that it can often be difficult for teams to know which ones to use and how they interact with one another. Also, studies have shown that, while many industrialists admit that the tools developed in academia would be beneficial to their NPD efforts, they claim that they carry a learning curve that is too steep and that they become too complex to manage over time. It is evident, then, that these tools are difficult to teach, especially to those unfamiliar with the NPD process. This research builds upon a streamlined process for the fuzzy front-end, improving the usability of the tools involved and making them accessible to everyone. The was achieved over the course of 2 years, following over 50 final year NPD teams from engineering, design, technology and construction as they carried a product from concept through to production specification. Questionnaires, focus groups and observations were used to understand the usability issues with the tools involved, and a human-centred design approach was adopted to produce a solution to these issues. The solution takes the form of a physical toolkit, similar to a board game, which allows the team to play through an example of a new product development in order to understand the process and the tools, before using it for their own product development efforts. A complimentary website is used to enhance the physical toolkit, and it provides more examples of the tools being used, as well as deeper discussions on each of the topics, allowing teams to adapt the process to their skills, preferences and product type. Students found the solution very useful and intuitive, and experienced significantly less confusion and mistakes with the process than teams who did not use it, claiming they would definitely use it in future projects outside of the university. Those with a design background found it particularly useful for engineering principles like Quality Function Deployment, while those with an engineering or technology background found it particularly useful for design and customer requirements acquisition principles like Voice of the Customer. Products developed using the toolkit are added to the website as more examples of how they can be used, creating a loop which helps future teams understand how the toolkit can be adapted to their project, whether it be a small consumer product or a large B2B service. The toolkit would serve as a useful aid when teaching students about the NPD process, as well as for educating those in industry, whether experienced companies or inexperienced start-ups.
\end{abstract}

Keywords: New product development, fuzzy front-end, usability, human-centred, UX design, Kano model, quality function deployment, voice of the customer

\section{INTRODUCTION}

New product development has always been a difficult practice as it is such a broad area, and there are so many stages, methods and variables. This is true for both real world application and in education. While there are many courses that focus on NPD theories, methodologies and case studies, it is evident that individuals need real-world, first-hand experience developing products in order to gain a strong grasp of the subject. This can be particularly challenging for courses where NPD is only taught in a single module, as there is not sufficient time for students to properly study all the stages of an NPD 
cycle, all the available methods for tackling these stages and how they differ across product types, or to apply this learning to a product development project. The problem is further complicated when students come from multiple course backgrounds and skillsets, like business, marketing or engineering and are expected to learn the same material. Topics like market research and customer requirements acquisition might feel familiar for marketing or design students but may appear alien to engineering or computer science students. Likewise, technical concepts like Quality Function Deployment will likely be easier for engineering students than for marketing or design students. However, students must be familiar with and respect these concepts if they are to work well together in interdisciplinary product development teams once they graduate.

The challenge of educating individuals on best NPD practices is constantly getting more difficult as the landscape continues to change. Due to sites like Netflix and Amazon offering personalised recommendations, as well as smart home devices adapting to each individual's unique needs and lifestyles, customers are increasingly expecting products tailored to suit them [1]. As enabling technologies like artificial intelligence and additive manufacturing rapidly continue to improve and become more accessible, many companies are rushing to figure out how they can be used to augment their current product offerings, or to offer new products and services. Meanwhile, the barriers to setting up a brand and selling a product are constantly being reduced, creating a strong growth in the popularity of start-ups and placing even more pressure on firms to innovate and compete for market share [2]. The end result is a product development landscape that is quickly becoming fast-paced, risky and demanding. It is evident that there is a strong need for a methodology that helps firms to tackle these problems, as well as a workforce educated in applying it.

O'Sullivan and Sheahan [3] have already developed a streamlined methodology for navigating the fuzzy front-end, adopting tools with strong synergy like Voice of the Customer (VoC), the Kano model and Quality Function Deployment (QFD). Carrying out this process allows teams to collect the information required to play a serious game called Buy a Feature, whereby potential customers work together to buy features of the product with a limited budget [4]. The overall methodology is designed to quickly allow teams to assess a product concept by knowing if customers are likely to buy it, how they are likely to spend their money, and which features should be prioritised in order to achieve this. This information can then be used to decide whether or not the concept is worth pursuing and can also provide guidelines for the rest of the product development. However, while the methodology has been proven effective, it utilises tools which have been proven to have poor usability, making them difficult to teach in education and lowering their adoption rate in industry [5]. The aim of this research, then, is to understand the usability issues with these tools and provide a solution that makes them easier for both students and industrialists to learn and use.

\section{UNDERSTANDING THE USABILITY ISSUES}

The above methodology was used by almost 30 teams of final year new product development students from design, engineering, technology and construction backgrounds as they brought a product concept from the idea stage through to proof-of-concept and production plan. Upon completion of the project, research was carried out to investigate the students' experiences with the process and the tools within it, in order to gain an insight into their usability issues. This was done through surveys, focus groups and observations of the final reports. As a detailed report of the findings would require a lengthier paper, an overview of the key insights is provided here.

Most teams got on reasonably well with the VoC, though some teams carried out surveys and questionnaires just for the sake of it, without really having a goal defined and questions designed accordingly. Design students carried out the VoC particularly well, as expected. Meanwhile, the majority of teams executed the Kano questionnaire poorly and so were unable to categorise requirements properly using the Kano table. Focus groups showed that this was due to a lack of fundamental understanding of the concept, as well as its potential benefits to the projects. Likewise, the majority of teams struggled with the first QFD matrix. Students exhibited a poor understanding of the difference between customer requirements and the technical requirements that would fulfil them, and experienced great confusion using the roof to understand the interaction between these technical requirements. Finally, while the majority of teams found Buy a Feature useful and understood the benefits of it, many teams expressed frustration with the website used for playing the game as it was not very intuitive for them nor the players. Some teams also calculated their budget incorrectly and, again, demonstrated poor understanding of the difference between customer requirements and technical requirements, i.e. features 
were often presented to customers as technical solutions that they might not understand, rather than how the solution would benefit them.

No team said they would use the entire methodology again in future projects inside or outside of university, though the majority of teams did express interest in certain elements of the process. For example, while designers found Kano and QFD particularly frustrating, some of them expressed interest in using Buy a Feature in future, if it were easier to use. Similarly, some of the engineering and technology students said they would potentially use QFD and VoC, respectively, in future work. It was clear that, while usability of the tools themselves was a big issue, an arguably bigger issue was that teams often did not understand why they were using a certain tool or the benefits it would have to their work. This meant that they approached the tools with a negative attitude, just using them to please their lecturer and so were less determined to figure out how to use them properly.

\section{DeVEloping a solution}

Once the usability issues were better understood, a human-centred design approach was adopted to develop a solution. First, a list of requirements was created to guide the solution. Next, the researchers brainstormed potential new requirements with some of the students and with other NPD experts, and these were then categorised into 'needs' and 'wants'. It was clear that users first needed to understand the goal of the process and how each tool would help them to reach that goal, before focusing on the usability of the tools themselves. This could be achieved by using colour coding to show how the output of one step would become the input for another. It also became clear that teams should experience a walkthrough of the process before trying to adapt it to suit their own product concept and skills. This would ideally be done with a worked example of another product, so that teams could experience the process of developing a new product before starting with their own concept.

It was decided that the first version of the solution would be physical as this would allow for quick iteration, but future refined versions would likely be software-based. Through quick iteration and testing, the solution eventually took a form similar to that of a board game. Each step of the process is represented by a 'tile' on the board, so users can see the end goal from the beginning, and see the logical path required to get there. Each tile has an overview of the step which it represents, e.g. QFD, what is required in order to complete the step, and what the output will be. The tools are also colour coded to show how the output of one tool becomes the input for another, so that users understand why they are completing that step and how it will benefit their project. Pawns are used to represent each user and to ensure that the team moves together and are only focused on the step upon which they are placed.

Once the users reach the final step of the board, they can use the information collected along the way to fill in the templates provided in order to play Buy a Feature with a group of their potential customers. Instructions and space for playing this game are provided on the flip side of the board. An example product is also included in the form of cards which correspond to each tile on the board. This allows users to get an overview of each step by reading the tiles but can look at the corresponding cards for that tile to see an example of how this step was completed for another product development. This way, teams can experience the process of developing a product before adjusting the process to fit their own product concept. A complimentary website is also provided and can be accessed by scanning the QR code on the board. This provides extra examples, as well as links to existing resources for each of the tools, like instructions for carrying out surveys and interviews, or tips for brainstorming. All materials were professionally branded and printed, boxes were purchased, plastic inserts were vacuum formed, and pawns were created through 3D printing and silicone moulding, giving the product a very professional finish. The product was named Playthrough.

\section{METHODOLOGY FOR TESTING}

The product was tested with 28 teams of final year students from design, engineering, technology and construction backgrounds as they brought a product concept from an idea through to proof-of-concept and production plan. Each team was provided with a copy of Playthrough and advised to first go through the process with the example product included in the box, before adapting the process to suit their own needs. Following completion of the project, surveys, focus groups and observations of the reports were carried out to learn about the students' experiences with Playthrough and each of the tools involved. These results would then be compared to the same study carried out the year before without Playthrough to see if the usability issues had been improved. Cross-analysis would be carried out to compare the results across course backgrounds and product types. 


\section{RESULTS}

\subsection{Surveys}

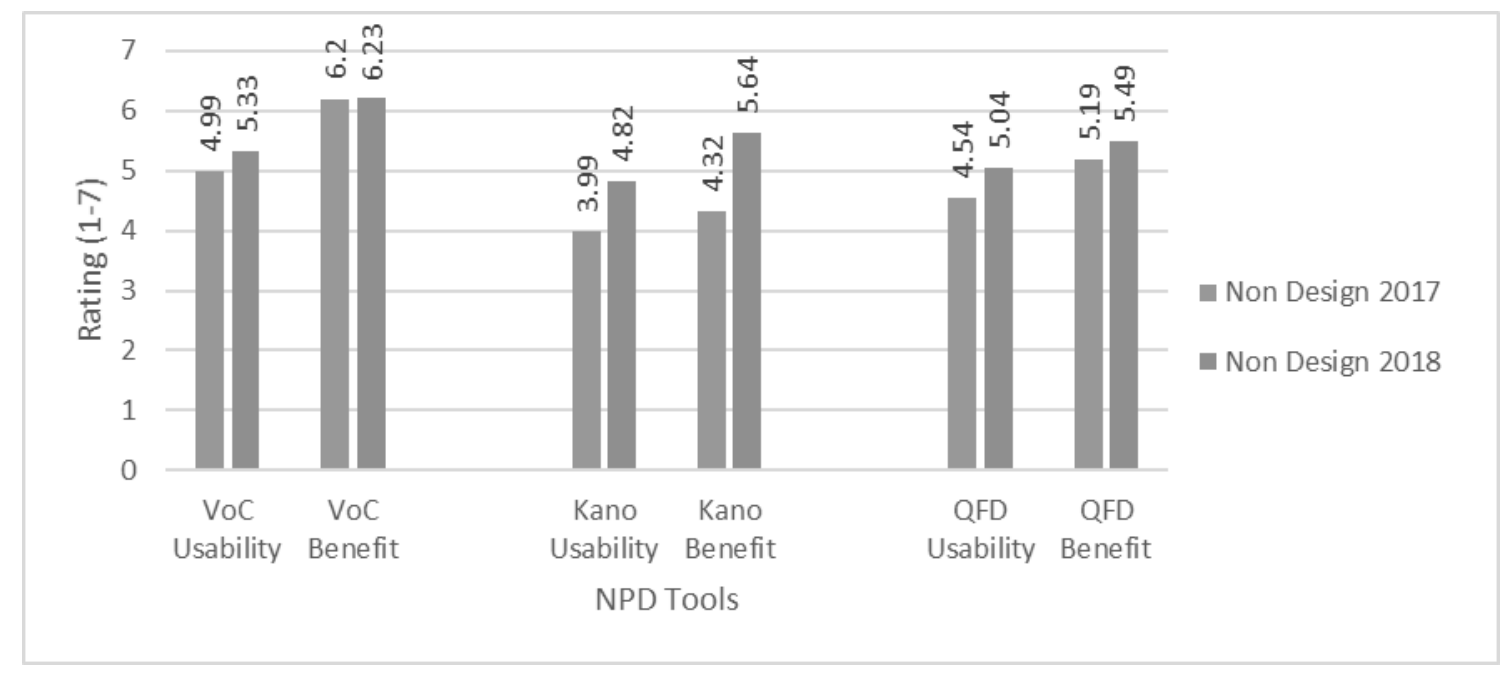

Figure 1. Comparison of non-design students' survey ratings of NPD tools between 2017 (without Playthrough) and 2018 (with Playthrough)

The graph compares survey results by non-design students between the 2017 study (without Playthrough) and the 2018 study (with Playthrough). Students were asked to rate the VoC, Kano and QFD in terms of their usability and the teams' initial understanding of how the tools would benefit their project. A scale of 1-7 was used, with 1 being 'very poor' and 7 being 'very good.'

\subsection{Observations}

Overall, teams who used Playthrough experienced far less confusion and errors than those who did not. The final project reports showed that teams who used Playthrough used almost every tool perfectly, except for a handful of teams who struggled with QFD because their product was too simple and had very few features, or because they were developing an app. Meanwhile, teams from the first study (where Playthrough was not used) used almost every tool incorrectly. These results are true for students across all courses.

\subsection{Focus Groups}

The overall responses from teams during the focus groups following the use of Playthrough were very positive, with teams expressing very little frustration with the process compared to the previous year. Those with a design background were less enthusiastic about the process than non-designers, with many claiming that they would rather use the processes they have been learning and using for years. Having said this, many designers still found some elements of the process very useful and said they would use them again in future projects, and that they could see how Playthrough would be very logical and helpful for those without a design background. Indeed, all non-designers said they would use the whole process again in future NPD efforts (See Figure 2).

While almost every team in the previous study complained about the usability of the Buy a Feature website, no team complained about the usability of it within Playthrough. Two teams did however mention that their product was small and cheap, and so the money provided was of too high value to play the game properly. 


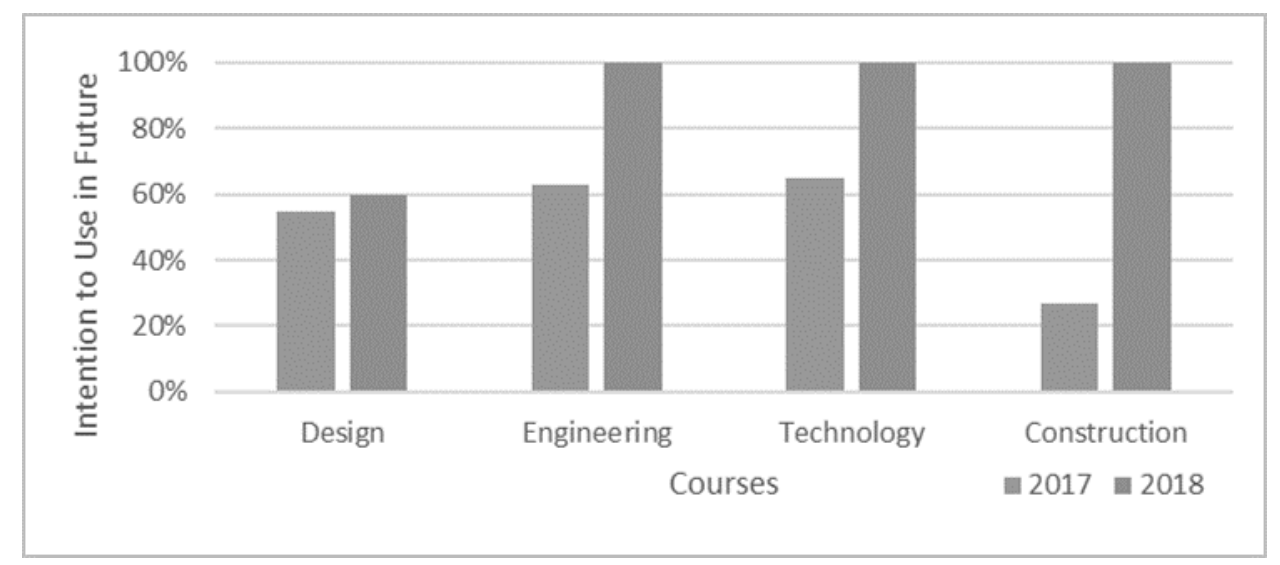

Figure 2. Comparison of teams' intentions to use the process in future NPD projects between 2017 and 2018 and across courses.

\section{DISCUSSION OF RESULTS}

\subsection{Surveys}

It was expected that the surveys would show a big improvement in the usability of the tools and students' initial understanding of how the tools would benefit their project, but the results were quite surprising. The results were not much different for students from a design background and were actually a slight disimprovement on the previous year. This might be because Playthrough forced users to go through each stage of the methodology and, like designers explained in the focus groups, they have studied various design processes for almost 4 years and have developed their own preferred methods. While Figure 1 does show an improvement for the non-design courses, it was not as much as expected. In the 2017 study without Playthrough, teams rated the tools quite poorly and the reports reflected this quite accurately as the majority of teams had not managed to use the tools correctly. In the 2018 study, however, almost all tools were used perfectly, but students still did not rate the tools very highly in the survey. This might be different if the students had first experienced how much more difficult and less intuitive the methodology would be without a solution like Playthrough. Indeed, many teams said during the focus groups that they experienced minor difficulties at first, but once they went through the example they quickly understood it.

\subsection{Observations}

From studying the reports, it was clear that there was a vast improvement in the application of the methodology over the previous year, as the majority of the teams used each tool almost perfectly. There was a huge improvement in the quality of the $\mathrm{VoC}$, as teams carried out effective online research, utilised the appropriate target market(s) for each product, used a mix of specific and open-ended questions in surveys, and used interviews and observations to dive deeper into specific areas. Teams executed the Kano Model very well, with the majority phrasing their questions perfectly and reaching out to large, appropriate sample sizes. In most cases, the requirements were categorised perfectly also. Following this, teams showed a drastic improvement in their use of QFD and demonstrated a strong understanding of how all the tools in the process worked together. While a small number of teams still struggled with the roof of the matrix, particularly in the cases of simple products with few features, almost all teams differentiated between customer and technical requirements well and multiplied out their results correctly. Overall, teams prioritised features a lot more clearly and confidently than teams in the previous year.

\subsection{Focus Groups}

The overall feedback during these group sessions was very positive, with most teams saying they found Playthrough very useful, intuitive and even enjoyable. The only negative comments came from some designers who said they would rather mix some of the tools with their own process, and the teams who developed either simple products or digital ones, as they struggled with QFD and Buy a Feature. However, they claimed that if more examples were added on how to use the methodology with these 
types of products, they would be happy to use it in future. One interesting, reoccurring point was that the toolkit should not be marketed as a 'game,' as this gave many teams the impression that it would be a fun game, rather than a methodology. Having said this, teams did appreciate the board-game-like approach to it, as it encouraged them to sit together and go through the steps collaboratively, rather than delegating tasks and working in silos. While it is also believed that the physical toolkit likely helped students learn better than they would from a screen, teams did say that an initial workshop or video giving an overview of Playthrough would be extremely useful.

\section{CONCLUSIONS, LIMITATIONS AND FUTURE WORK}

This research set out to understand the usability issues with tools used for navigating the fuzzy frontend and to design a solution to overcome these issues, and it has achieved this goal. While there are definite usability issues with tools like Kano and QFD, an arguably bigger problem is that teams often do not understand why they are using these tools or how they will benefit them, and so they do not put their full effort into figuring them out. Playthrough provides a solution to these problems by walking users through the fuzzy front-end in an intuitive and enjoyable way with an example product, before helping them to develop their own product. By using a board game analogy, teams can quickly understand the final goal of the process, and how each step links up to get them there. The colour coding of the tools and simple language for each step, combined with a website that provides more detailed information when needed, has proven effective in educating final year students on a large portion of the NPD process. Student teams that used Playthrough used almost every tool perfectly, whereas in the previous study, not one team managed to use each tool correctly. This resulted in teams having a much stronger understanding of their customers' requirements, the features they would use to satisfy these requirements, and how they would prioritise these features going fourth into the design stage. The solution led to a dramatic increase in students' intentions to use the methodology again in future NPD efforts, both inside and outside of university. Designers were still less enthusiastic about this methodology than the ones they are already used to but said they would pick and adapt various steps from the process to integrate into their own. Having said this, it is interesting to note that, in a peer assessment at the end of the project, 9 out of the top 10 designs came from a non-design background, and this module is usually dominated by the designers. One possible limitation to the study could be the breadth of products designed. One team in the second study claimed the VoC was difficult and rated it lowly in the survey, though it became evident during the focus groups that this was because they were designing a product for Parkinson's sufferers and so experienced difficulty finding a large sample size for their VoC. Another team experienced difficulty with QFD as they were designing an app, while the product example included with Playthrough was a physical one. Future studies should aim to add these products to the website as more examples to help future students adapt the process to suit their needs, and as an incentive to produce good work.

\section{REFERENCES}

[1] Franke N., Keinz P. and Steger C.J., Testing the value of customisation: when do customers really prefer products tailored to their preferences? Journal of marketing. 2009 Sep 1;73(5):10321.

[2] Zwilling M. A New Era for Entrepreneurs and Start-ups Has Begun. Available: https://www.forbes.com/sites/martinzwilling/2013/12/25/a-new-era-for-entrepreneurs-andstartups-has-begun/\#58c0ba754bd1 [Accessed 2019, 13 Feb] (2013, 25 Dec). Forbes.

[3] O'Sullivan M.N. and Sheahan C. Using Serious Games to Guide Commercial Success from the Fuzzy Front-End. In European Conference on Innovation and Entrepreneurship, ECIE'13, Portugal, September 2018, pp.1081-1084.

[4] Hohmann L. Innovation games: creating breakthrough products through collaborative play, 2006 (Pearson Education).

[5] Thia C.W., Chai K.H., Bauly J. and Xin Y. An exploratory study of the use of quality tools and techniques in product development. The TQM Magazine, 2005, 17(5), 406-24. 